\title{
Mechanisms for Photon-Emission Enhancement with Silicon Doping in InGaN/GaN Quantum-Well Structures
}

\author{
YUNG-CHEN CHENG, ${ }^{1}$ CHENG-HUA TSENG, ${ }^{2}$ CHEN HSU, ${ }^{2}$ \\ KUNG-JEN MA, ${ }^{3}$ SHIH-WEI FENG,${ }^{4}$ EN-CHIANG LIN,${ }^{4}$ C.C. YANG,${ }^{4,6}$ \\ and JEN-INN CHYI ${ }^{5}$ \\ 1.-Graduate Institute of Electro-Optical Engineering, National Taiwan University, Taipei, Taiwan, \\ Republic of China. 2.-Department of Mechanical Engineering, Chung Cheng Institute of Tech- \\ nology, National Defense University, Tahsi, Taoyuan, Taiwan, Republic of China. 3.-Department of \\ Mechanical Engineering, Chung Hua University, Hsinchu, Taiwan, Republic of China. 4.-Graduate \\ Institute of Electro-Optical Engineering, Department of Electrical Engineering and Graduate Insti- \\ tute of Electronics Engineering, National Taiwan University, Taipei, Taiwan, Republic of China. \\ 5.-Department of Electrical Engineering, National Central University, Chung-Li, Taiwan, Republic \\ of China. 6.—E-mail: ccy@cc.ee.ntu.edu.tw
}

Material and optical analyses of three InGaN/GaN quantum-well (QW) samples with different silicon-doping conditions were conducted. Quantum-dot (QD) structures were observed in samples of silicon doping either in barriers or wells. The calibrated-radiative lifetimes in both silicon-doped samples showed the consistent trend of the formation of zero-dimensional (0-D) structure upon silicon doping. In optical characterization, the barrier-doped sample showed a blueshift of the photoluminescence (PL) peak, enhancement of integrated PL intensity, reduction of Stokes shift (SS), decrease of carrier-activation energy, and shortening of PL decay time. Except the insignificant PL peak shift, the well-doped sample showed similar trends, although they are not as prominent as the barrier-doped sample. Such results mainly originated from the reduction of the quantum-confined Stark effect (QCSE) within the clusters. Contrary to the interpretation in the past, the major mechanism for QCSE reduction is due to strain relaxation, instead of carrier screening, in conjunction with the formation of QD structures. Such a conclusion is supported by the result of smaller changes of optical behavior in the well-doped sample, in which carrier screening is expected to be more significant. In this sample, besides strain relaxation, enhanced carrier localization (CL) might represent another important mechanism for photon-emission improvement.

Key words: Photon emission, silicon doping, quantum-well structures, InGaN/GaN

\section{INTRODUCTION}

Silicon doping in InGaN/GaN quantum-well (QW) structures has been an issue of great interest for improving photon-emission efficiency. Typically, with increasing silicon-doping concentration, particularly doping in barriers, the following phenomena were observed: (1) the photoluminescence (PL) peak blue shifted, (2) the Stokes shift (SS) decreased, (3) the PL decay time reduced, and (4) the PL intensity increased. ${ }^{1-6}$ Such variations in optical properties were usually attributed to the screening of the quantum-confined Stark effect (QCSE) ${ }^{3-5}$ because of the doped carriers. In other words, the piezoelectric field was effectively reduced with silicon doping

(Received August 15, 2002; accepted November 13, 2002) through carrier screening. Also, carrier localization (CL) has been discussed for interpreting optical characterization results of barrier-doped samples. ${ }^{7,8}$ Based on their optical measurements, some research groups claimed that potential fluctuation and, hence, CL was reduced in silicon-doped InGaN/GaN QW structures. ${ }^{2,9}$ Meanwhile, nanoscale islands (500 nm in size) in such a sample were hypothesized based on atomic force microscopy and cathodoluminescence measurements. ${ }^{9}$ In addition, sharp layer interfaces were speculated based on $\mathrm{x}$-ray diffraction data. ${ }^{10,11}$ With all these efforts, however, the real material microstructures and their connections with optical properties in silicon-doped InGaN/GaN QW structures have never been well discussed. Information of material microstructures can provide direct evidence for interpreting the results of optical characterization. 
Based on the observations in material analysis, our research emphasized the microstructure alterations with silicon doping and their connections with optical properties. In particular, the influences of induced-strain relaxation and carrier confinement on optical characteristics were studied. The results of barrier and well-doped samples are compared to understand their differences. For these purposes, we performed PL, photoluminescence excitation (PLE), time-resolved photoluminescence (TRPL), and high-resolution transmission electron microscopy (HRTEM) to understand the dominating mechanisms that contribute to the improved photon-emission efficiency in InGaN/GaN multiple QWs with a silicondoping concentration at $5 \times 10^{18} \mathrm{~cm}^{-3}$. The results show that samples with silicon doping tend to form zero-dimensional (0-D) clusters, i.e., quantum dots (QDs). Consequently, strain relaxation, caused by microstructure alteration upon silicon doping, may play a key role in improving photon-emission efficiency, particularly in a barrier-doped sample. On the other hand, the enhanced-carrier confinement may be the major cause for increasing photon-emission intensity in a well-doped sample.

In this paper, the procedures of sample preparation and experiments are discussed. Next, the results of material analysis, i.e., HRTEM results, are presented. Then, various optical characterization data are reported. Discussions for interpreting the measurement results are given. Finally, conclusions are drawn.

\section{SAMPLE PREPARATION AND EXPERIMENTAL PROCEDURES}

Three InGaN/GaN QW samples of the same geometry and nominal-indium content were prepared with metal-organic chemical-vapor deposition growth on a sapphire substrate. They all consisted of five QW periods with $3 \mathrm{~nm}$ in well width and $7 \mathrm{~nm}$ in barrier width. A 20-nm GaN-cap layer was grown on top of the five $\mathrm{QW}$ layers. The nominal-indium content was about $15 \%$. The growth temperatures were $1,050^{\circ} \mathrm{C}$ and $740^{\circ} \mathrm{C}$ for $\mathrm{GaN}$ and InGaN, respectively. The doping concentrations are the same $\left(5 \times 10^{18} \mathrm{~cm}^{-3}\right)$ either in barriers or in wells. The undoped, barrierdoped, and well-doped samples are referred to as samples A, B, and C, respectively. Continuous-wave (CW)-PL measurements were carried out with the 325-nm line of a 35-mW He-Cd laser. The samples were placed in a cryostat for temperature-dependent measurements ranging from 10-300 K. The PLE experiments were conducted using a quasi-monochromatic excitation-light source from a xenon lamp dispersed by a $0.5-\mathrm{m}$ monochromator. The emission wavelength of PLE measurements was set at the peak energies of individual CW-PL spectra.

The TRPL measurements were performed using a Hamamatsu streak camera (Hamamatsu Photonics $\mathrm{KK}$, Japan) in conjunction with a 25-cm monochromator using a 600 lines/mm grating. The temporal resolution of the streak camera was about $5 \mathrm{ps}$. The excitation pulses were obtained from the second-harmonic generation of a mode-locked Ti:sapphire laser with a pulse width of $100 \mathrm{fs}$ and a repetition rate of $76 \mathrm{MHz}$. The excitation wavelength, spot size, and power were $390 \mathrm{~nm}$, about $2 \mathrm{~mm}$ in diameter, and $60 \mathrm{~mW}$, respectively. The HRTEM investigations were conducted with a $200-\mathrm{KeV}$ Philips CM 200 and a $300-\mathrm{KeV}$ JEM 3010 microscope (Philips, The Netherlands). All the high-resolution micrographs were taken at Scherzer defocus, and the sample was viewed along a [11-20] zone axis. The $300-\mathrm{KeV}$ JEM 3010 microscope was equipped with a $2 \mathrm{k} \times 2 \mathrm{k}$ slow-scan charge-couple device camera and Gatan imaging filter.

\section{MATERIAL ANALYSIS RESULTS}

Figures 1-3 show the HRTEM images of samples $\mathrm{A}, \mathrm{B}$, and C, respectively. Although the five QWs can be clearly identified in Fig. 1 (sample A, undoped), the image here indicates that indium composition extensively spreads from wells into barriers to form clusters of quite large and irregular sizes. On the contrary, as shown in Fig. 2, in the barrier-doped sample (B), nanoscale clusters (QDs) with more regular sizes and shapes are arranged along the designated QW layers. The sizes of the dots range from

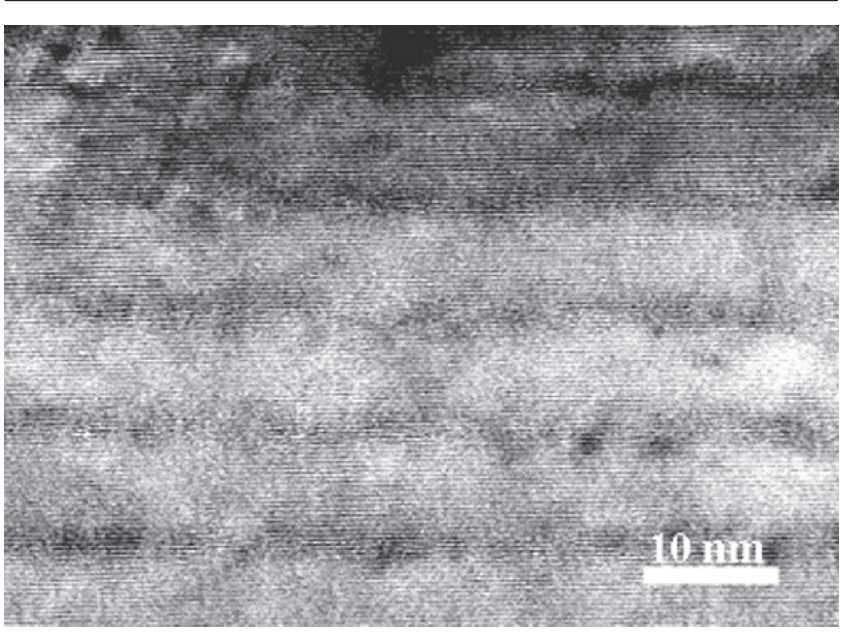

Fig. 1. The HRTEM image of sample A.

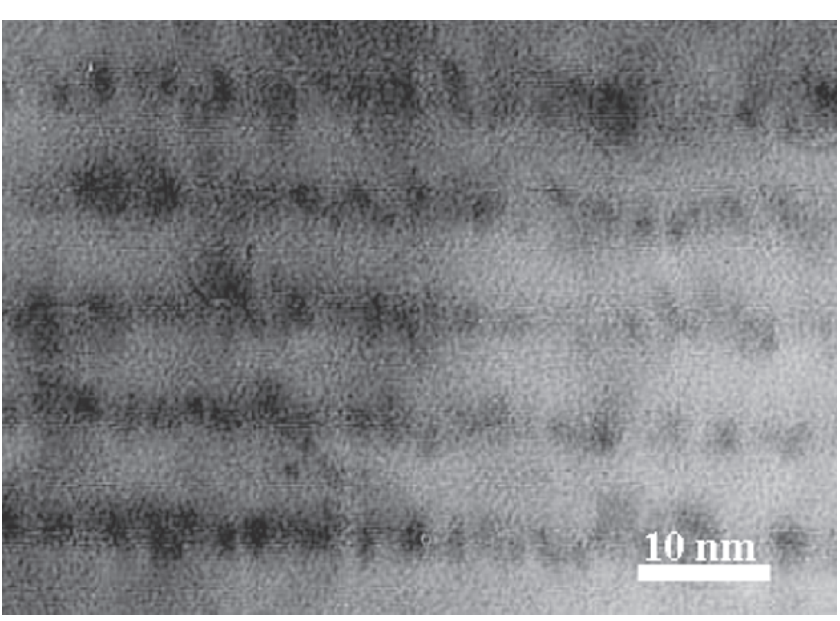

Fig. 2. The HRTEM image of sample B. 


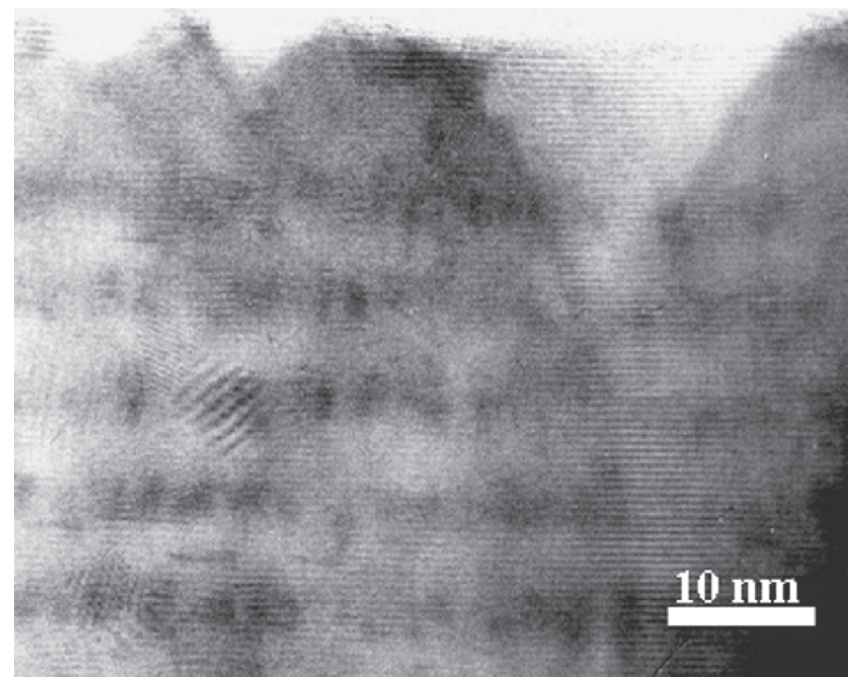

Fig. 3. The HRTEM image of sample C.

3-5 nm. Such structures are expected to strongly localize excitons, preventing nonradiative recombination outside clusters. Although the quasi-regular arrays of QD in the well-doped sample (C) is not as clear as the barrier-doped sample, sharp clusters can be clearly seen in sample C (Fig. 3). In Fig. 3, a V-shaped defect and a threading dislocation can be observed on the right-hand side of the image. Also, a few fringe patterns, which represent phase-separated clusters, can also be seen at the lower left corner of the image. Usually, it is believed that the density of indiumaggregated and phase-separated clusters is higher nearby a threading dislocation. ${ }^{12}$ From the optical and material analyses of similar samples in other research, ${ }^{13}$ we learned that the $\mathrm{QD}$ structures in samples B and C typically result in higher photonemission efficiencies, which are to be confirmed in the following discussions of optical characterization.

\section{OPTICAL CHARACTERIZATION RESULTS}

Figure 4 shows the PL spectral-peak positions as functions of temperature of the three samples. The blueshift ( $73 \mathrm{meV}$ at $10 \mathrm{~K}$ ) of the PL peak in sample B compared with the undoped sample (A) has been widely observed. $^{3-5}$ The PL peaks of the well-doped sample (C) approximately coincide with those of the undoped sample (A), except the small redshifts below $80 \mathrm{~K}$ and above $200 \mathrm{~K}$. All the curves in Fig. 4 show S-shaped variations of the PL peak, which is typical behavior of such clustered and strained structures.

Figure 5 shows the temperature-dependent, integrated PL intensities of the three samples. One can see that silicon doping results in higher photonemission efficiencies, particularly in the temperature range above $200 \mathrm{~K}$. With silicon doping, the normalizedluminescence intensity is about two orders of magnitude higher than the undoped sample at room temperature. It is interesting to note that the normalized, integrated PL intensity variations with temperature of all the samples can generally be divided

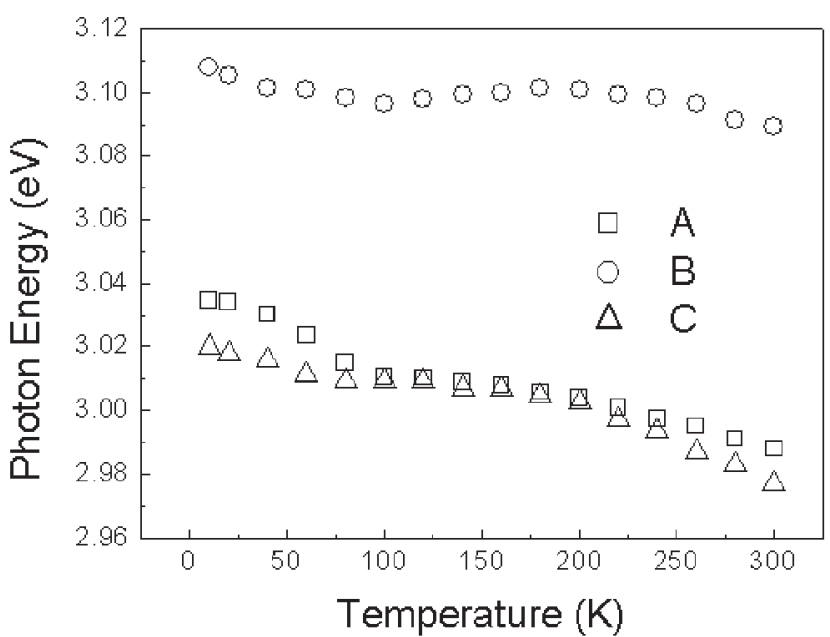

Fig. 4. The PL spectral-peak positions versus temperature of the three samples.

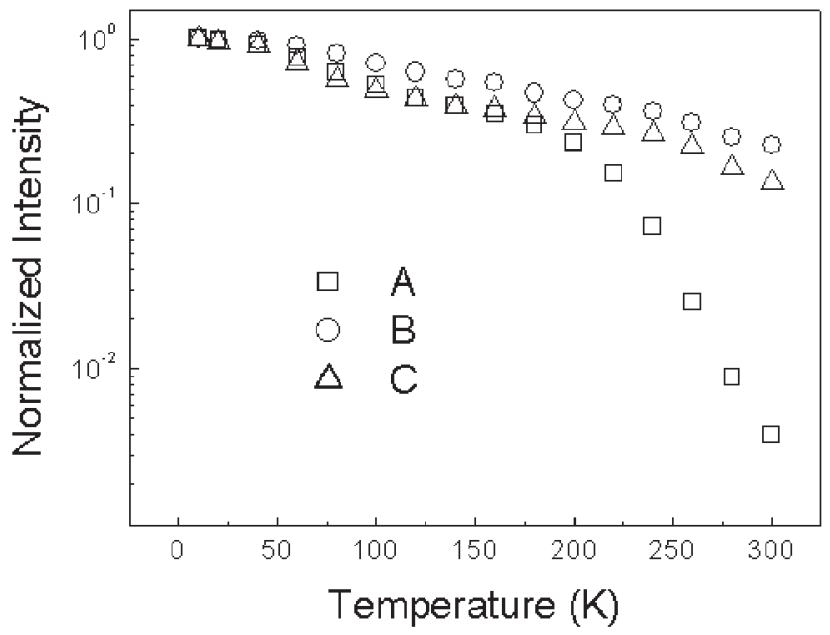

Fig. 5. The normalized integrated PL intensities versus temperature of the three samples.

into three stages with the boundaries around $80 \mathrm{~K}$ and $200 \mathrm{~K}$.

The Arrhenius plots of the integrated PL intensity as a function of inverse temperature are shown in Fig. 6. Here, $\mathrm{k}$ is Boltzman's constant. The solid lines in Fig. 6 illustrate the best-fitting curves by using an equation of the form ${ }^{14}$

$$
\begin{gathered}
\mathrm{I}(\mathrm{T})=\mathrm{A}_{0}\left[1+\mathrm{B}_{0} \exp \left(-\mathrm{E}_{1} / \mathrm{kT}\right)\right. \\
\left.+\mathrm{C}_{0} \exp \left(-\mathrm{E}_{2} / \mathrm{kT}\right)\right]^{-1}
\end{gathered}
$$

Here, I(T) is the temperature-dependent, normalized integrated PL intensity. The two activation energies, $\mathrm{E}_{1}$ and $\mathrm{E}_{2}$, correspond to two different decay processes. The notations $\mathrm{B}_{0}$ and $\mathrm{C}_{0}$ represent the ratios of the probability of nonradiative to that of radiative transitions. The constant $\mathrm{A}_{0}$ is a fitting parameter. In the low-temperature range, the small decrease in PL intensity is dominated by the first exponential factor with a small activation energy, $\mathrm{E}_{1}$, ranging from $12 \mathrm{meV}$ to $18.1 \mathrm{meV}$ (the legends of 


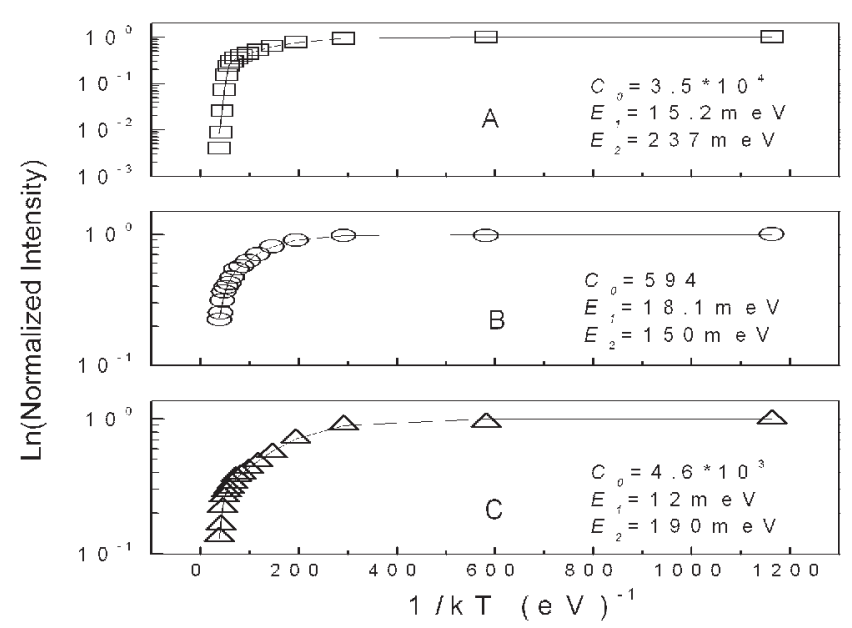

Fig. 6. The Arrhenius plots of the integrated PL intensity as a function of temperature.

Fig. 6) and a constant $B_{0}$ of order 10 for all samples. This portion of decay is supposed to originate from carrier transport within a cluster or between coupled clusters and carrier consumption by the defects there. In fitting the high-temperature behavior, the constant, $\mathrm{C}_{0}$, of $3.5 \times 10^{4}$ in the undoped sample was calibrated. The $\mathrm{C}_{0}$ values of samples $\mathrm{B}$ and $\mathrm{C}$ are reduced to 594 and $4.6 \times 10^{3}$, respectively. Also, the second activation energies, $\mathrm{E}_{2}$, are changed from $237 \mathrm{meV}$ of sample A to $150 \mathrm{meV}$ of sample B and $190 \mathrm{meV}$ of sample C. The major mechanisms for the reductions of constant $\mathrm{C}_{0}$ and the corresponding activation energy, $\mathrm{E}_{2}$, of samples $\mathrm{B}$ and $\mathrm{C}$ can be different. Figure 7 shows the PLE and CW-PL spectra of the three samples at $10 \mathrm{~K}$. Different SSs among the three samples can be clearly seen. The reductions of SS in samples B and C (reduced from that of sample A) were usually connected with the decrease of piezoelectric field. ${ }^{15}$ As shown in Fig. 7, the reduction of strain field is more significant in the barrierdoped sample.

The PL decay times at various emission-photon energies at $10 \mathrm{~K}$ are shown in Fig. 8. In most cases of similar measurements, the TRPL intensity shows a (at least) two-component decay profile when the photon energy is above a certain level or the sample temperature is above a certain value. ${ }^{16}$ Such a multicomponent-decay profile was observed in sample A but not in samples B and C. The empty symbols in Fig. 8 show the decay times of the extended-decay ranges. The filled symbols (only for sample A) stand for the decay times of the earlystage faster decay range. The overall decrease of extended-decay time in silicon-doped samples can be clearly seen. To help in interpretation, timeintegrated PL spectra based on TRPL measurements are also plotted. Different photon-energy dependencies of PL decay time among the three samples can be clearly seen.

Figure 9 shows the temperature-dependent (extended) decay-time variations at individual PL peaks of the three samples. The reduction of PL decay time

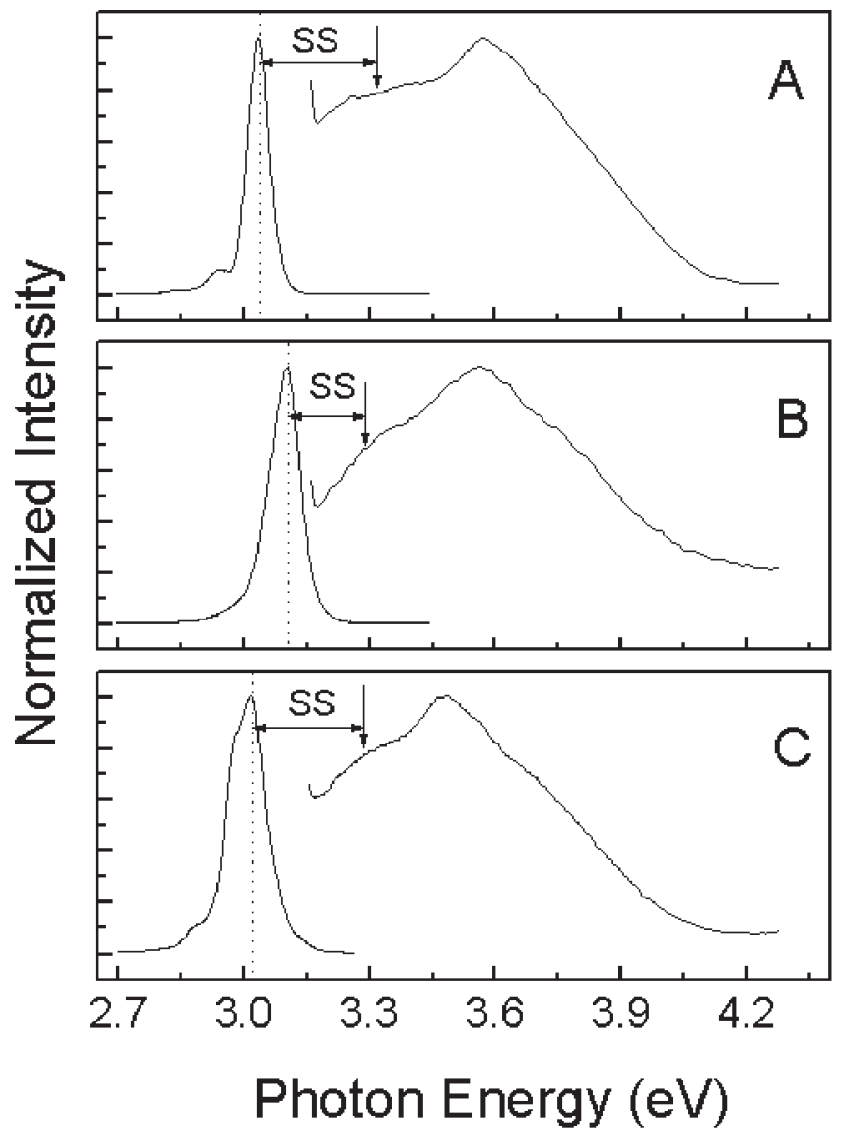

Fig. 7. The PL and PLE spectra at $10 \mathrm{~K}$ of the three samples.

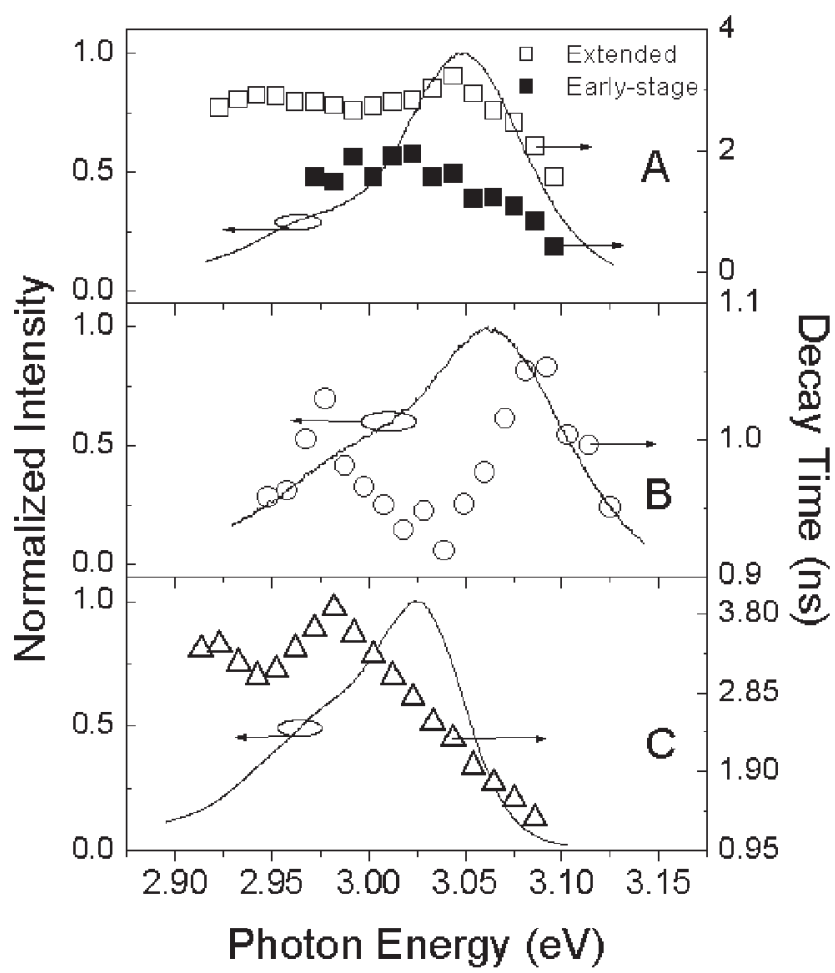

Fig. 8. The PL decay times at various emission-photon energies with the empty symbols for extended-decay times and filled symbols for early-stage fast decay times. 


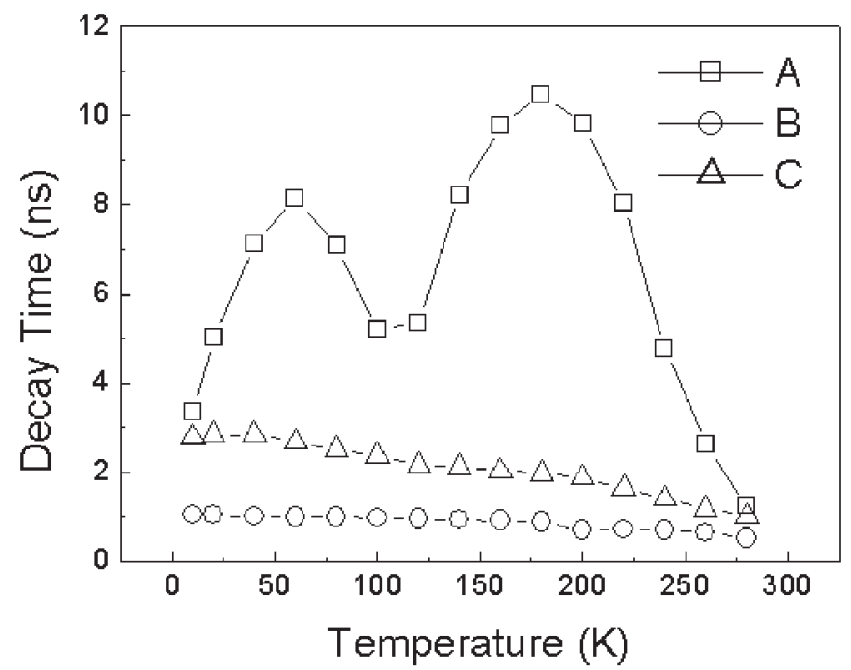

Fig. 9. The PL decay times at individual PL peaks versus temperature of the three samples.

upon silicon doping is more significant in the barrierdoped sample than the well-doped one. Two peaks with temperature variation can be clearly seen in sample A. Such peak features become less prominent in samples $\mathrm{B}$ and $\mathrm{C}$. The two peaks of PL decay time appear at $60 \mathrm{~K}$ and $180 \mathrm{~K}$ (denoted by $\mathrm{T}_{1}$ and $\mathrm{T}_{2}$, respectively), which are roughly consistent with the counterparts in Fig. 5, implying that they have certain physical meanings.

Figure 10 shows the radiative lifetimes as functions of temperature of the three samples. The radiative lifetimes, $\tau_{\text {rad }}(T)$, were calibrated according to the following equation: ${ }^{17}$

$$
\tau_{\text {rad }}(\mathrm{T})=\tau_{\mathrm{L}}(\mathrm{T}) \times \mathrm{I}_{0} / \mathrm{I}(\mathrm{T})
$$

Here, $I(T)$ is the temperature-dependent integrated $\mathrm{PL}$ intensity, and $\mathrm{I}_{0}$ is that at a low temperature $\left(10 \mathrm{~K}\right.$ in our calibration). Also, $\tau_{\mathrm{L}}(\mathrm{T})$ is the temperature-dependent (extended) PL decay time. The temperature-dependent radiative-lifetime variation has an important indication of material microstructure. Theoretically, radiative lifetime can be expressed as ${ }^{18}$

$$
\tau_{\text {rad }}(T) \propto T^{\alpha}
$$

Here, $\alpha$ is the dimension number of exciton-free motion multiplied by $1 / 2$. In a QD (QW) structure, $\alpha$ should be 0 (1). In a bulk material, $\alpha$ is $3 / 2$. From the results in Fig. 10, it is difficult to tell for sure that the samples have 0-D-, two-dimensional-, or threedimensional-like structures. However, the trend clearly indicates that the structures of silicon-doped samples are more 0-D-like than the undoped sample, particularly in the barrier-doped sample. This trend is consistent with the comparison results between Figs. 1, 2, and 3.

\section{DISCUSSION}

Indium-composition distribution in an InGaN/GaN QW sample is controlled with the processes of strain-

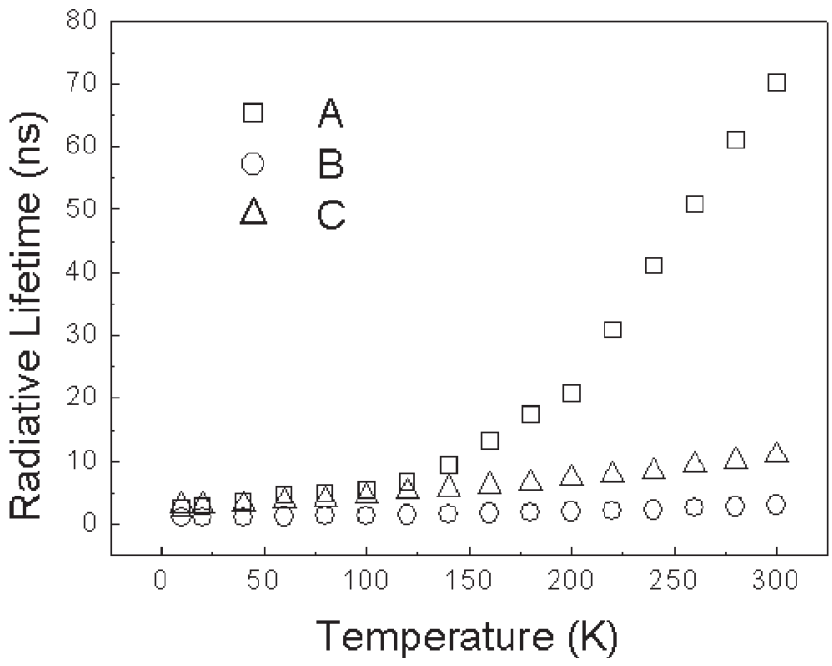

Fig. 10. The radiative lifetimes versus temperature of the three samples.

induced island formation and spinodal decomposition. ${ }^{19,20}$ Such processes are determined by the strain energy built in the QW layers. The HRTEM image in Fig. 1 for sample A shows a typical structure of indium-composition fluctuation. Although further studies are still required, it is usually believed that the process of $\mathrm{QD}$ formation has a strong connection with strain-energy relaxation in the material system. With silicon doping in barriers or wells, strains could be relaxed. ${ }^{21}$ The strain relaxation in QWs may have the effect of enhancing QD formation through spinodal decomposition or other strainrelated processes. Such a process seems to be more effective in the barrier-doped sample, as evidenced with the comparison between Figs. 2 and 3.

With the strain relaxed and the quasi-regularly arranged QDs formed, QCSE within dotlike clusters is reduced, leading to a PL blueshift and an enhanced radiative-transition rate. The later leads to a PL intensity increase and PL decay time reduction. Also, SS is expected to be reduced. These predictions have been confirmed with the results shown in Figs. 4, 5, and 7-9. In the past, the abovementioned optical phenomena observed in barrier silicon-doped samples were usually attributed to carrier screening of the piezoelectric fields upon silicon doping. ${ }^{3-5}$ However, the comparison between the results of samples B and $\mathrm{C}$ indicates that the carrier-screening effect may play a minor role in reducing QCSE in our samples. This is because the reduction of QCSE is less significant in the well-doped sample, in which more carriers are expected in the designated well layers (and inside the clusters) for piezoelectric-field screening. Therefore, although we observed similar optical properties as previously reported, the results must be reinterpreted. The significant blueshift of the PL peak in sample B (Fig. 4) can be attributed to the effect of quantum confinement besides the reduction of QCSE. The insignificant shift of the PL peak in sample C may originate from the interplay of sev- 
eral different mechanisms, including the reduction of QCSE (blueshift trend) and the indium-clustering effect (redshift trend). In the process of further indium clustering upon silicon doping, carrier potential is reduced, and carriers are more strongly localized. This mechanism may dominate in sample C. The formation of QDs means that the potential fluctuation becomes less randomly distributed. This is another reason for the reduction of SS in the doped samples, particularly in sample B.

The characteristic temperatures of $T_{1}$ and $T_{2}$ for dividing the stages of temperature-dependent variations of integrated PL intensity and PL decay time were often observed in similar structures in our research, particularly in undoped samples. Below $\mathrm{T}_{1}$, carriers can move around near the center of a cluster and relax into the potential minimum such that the PL decay time increases, and the PL intensity almost keeps constant with increasing temperature. Between $\mathrm{T}_{1}$ and $\mathrm{T}_{2}$, carriers receive certain thermal energy. Hence, they can move essentially freely within a cluster or transport between coupled clusters. In this situation, carriers may be consumed through defects, which are existent around clusters, such as stacking faults and local distortions. Therefore, PL intensity decreases with a certain slope. Also, PL decay time may first decrease because of the aforementioned defects. However, with more thermal energy, carriers can overcome certain barriers and be trapped by more strongly localized states. Hence, the PL decay time increases again. Beyond $\mathrm{T}_{2}$, carriers can escape from localized states, and PL intensity decreases more significantly with temperature. Also, PL decay time decreases monotonically with temperature. With silicon doping, more discrete QDs are formed, and carrier transport between them for stronger localization becomes more difficult. Hence, the peak features of PL decay time become less significant (Fig. 9).

The fitting results of the Arrhenius plots in Fig. 6 deserve further discussion. The activation energy, $\mathrm{E}_{1}$, can be regarded as that for carriers to overcome and then move between localized states of different potential levels. In this process, the three samples have about the same results. Nevertheless, the behaviors related to the second activation energy, $\mathrm{E}_{2}$, are quite different among the three samples. Such behaviors represent another key result of microstructure alteration upon silicon doping. The smaller $\mathrm{E}_{2}$ and $\mathrm{C}_{0}$ of sample B strongly indicate the relaxation of the strain field. The smaller $\mathrm{E}_{2}$ can also be attributed to the strong quantum confinement in this sample. On the contrary, the relative larger $\mathrm{E}_{2}$ and $\mathrm{C}_{0}$ of sample $\mathrm{C}$ reveal that quantum confinement and the reduction of QCSE in this sample are not as significant as sample B. The significant enhancement of integrated PL intensity in sample $\mathrm{C}$ can be attributed to the increase of carrier localization caused by more regular cluster structures.

\section{CONCLUSIONS}

In summary, we have conducted material and optical analyses of three InGaN/GaN QW samples with different silicon-doping conditions. From HRTEM images, QDs were observed in samples of silicon doping either in barriers or wells. The calibratedradiative lifetimes in both silicon-doped samples showed the consistent trend of the formation of $0-D$ structures upon silicon doping. In particular, clear QD arrays could be seen in the barrier-doped sample. In optical characterization, the barrier-doped sample showed a blueshift of the PL peak, enhancement of the integrated PL intensity, reduction of the SS, decrease of the carrier-activation energy, and shortening of the PL decay time. Except the insignificant PL peak shift, the well-doped sample showed similar trends, although they were not as prominent as the barrier-doped sample. Such results mainly originated from the reduction of QCSE within the clusters. Although carrier screening has been used for explaining the reduction of QCSE, it was believed that the major mechanism for QCSE reduction was due to strain relaxation in conjunction with the formation of QD structures. Such a conclusion was supported by the results of smaller changes of optical behavior in the well-doped sample, in which carrier screening was expected to be more significant. In this sample, the major mechanism for photonemission improvement might be the enhanced carrierlocalization effect.

\section{ACKNOWLEDGEMENTS}

This research was supported by the National Science Council, The Republic of China, under Grant Nos. NSC 90-2112-M-002-052, NSC 90-2215-E-002027, and NSC 90-2215-E-002-041.

\section{REFERENCES}

1. M.S. Minsky, S. Chichibu, S.B. Fleischer, A.C. Abare, J.E. Bowers, E.L. Hu, S. Keller, U.K. Mishra, and S.P. DenBaar, Jpn. J. Appl. Phys. 37, L1362 (1998).

2. J. Dalfors, J.P. Bergman, P.O. Holtz, B.E. Sernelius, B. Monemar, H. Amano, and I. Akasaki, Appl. Phys. Lett. 74, 3299 (1999).

3. E. Oh, C. Sone, O. Nam, H. Park, and Y. Park, Appl. Phys. Lett. 76, 3242 (2000).

4. M.Y. Ryu, P.W. Yu, E.J. Shin, J.I. Lee, S.K. Yu, E. Oh, O.H. Nam, C.S. Sone, and Y.J. Park, J. Appl. Phys. 89, 634 (2001).

5. C.K. Choi, Y.H. Kwon, B.D. Little, G.H. Gainer, J.J. Song, Y.C. Chang, S. Keller, U.K. Mishra, and S.P. DenBaars, Phys. Rev. B 64, 245339-1 (2001).

6. S. Chichibu et al., Appl. Phys. Lett. 73, 496 (1998).

7. T. Deguchi, A. Shikanai, K. Torii, T. Sota, S. Chichibu, and S. Nakamura, Appl. Phys. Lett. 72, 3329 (1998).

8. T. Wang, H. Saeki, J. Bai, T. Shirahama, M. Lachab, S. Sakai, and P. Eliseev, Appl. Phys. Lett. 76, 1737 (2000).

9. K. Uchida, T. Tang, S. Goto, T. Mishima, A. Niwa, and J. Gotoh, Appl. Phys. Lett. 74, 1153 (1999).

10. Y.H. Cho, F. Fedler, R.J. Hauenstein, G.H. Park, J.J. Song, S. Keller, U.K. Mishra, and S.P. DenBaars, J. Appl. Phys. 85, 3006 (1999).

11. Y.H. Cho, J.J. Song, S. Keller, M.S. Minsky, E. Hu, U.K. Mishra, and S.P. DenBaars, Appl. Phys. Lett. 73, 1128 (1998). 
Mechanisms for Photon-Emission Enchancement

12. Y.S. Lin, K.J. Ma, C. Hsu, S.W. Feng, Y.C. Cheng, C.C. Liao, C.C. Yang, C.M. Lee, and J.I. Chyi, Appl. Phys. Lett. 77, 2988 (2000)

13. Y. S. Lin et al., Appl. Phys. Lett. 80, 2571 (2002).

14. R. Seitz, C. Gaspar, T. Monteiro, E. Pereira, M. Leroux, B. Beaumont, and P. Gibart, J. Cryst. Growth 189/190, 546 (1998).

15. H.C. Yang, T.Y. Lin, and Y.F. Chen, Appl. Phys. Lett. 78, 338 (2001).

16. S.W. Feng, Y.Y. Chung, C.W. Liu, Y.C. Cheng, C.C. Yang, M.H. Mao, Y.S. Lin, K.J. Ma, and J.I. Chyi, Appl. Phys. Lett. 80, 4375 (2002)
17. R.C. Miller, D.A. Kleinman, W.A. Nordland, Jr., and A.C. Gossard, Phys. Rev. B 22, 863 (1980).

18. J. Feldman, G. Peter, E.O. Gobel, P. Dawson, K. Moore, C. Foxon, and R.J. Elliot, Phys. Rev. Lett. 59, 2337 (1987).

19. J.W. Cahn, Acta Metall. 10, 179 (1962).

20. H. Ho and G.B. Stringfellow, Appl. Phys. Lett. 69, 2701 (1996).

21. S. Ruvimov, Z. Liliental-Weber, T. Suski, J.W. Ager III, J. Washburn, J. Krueger, C. Kisielowski, E.R. Weber, H. Amano, and I. Akasaki, Appl. Phys. Lett. 69, 990 (1996). 